\title{
Present and future of solar neutrino physics
}

\author{
Vito Antonelli* ${ }^{\dagger}$ \\ Dipartimento di Fisica, Università degli Studi di Milano e I.N.F.N. Sezione di Milano, Via \\ Celoria 16 - 20133 MIlano, Italy E-mail: vito.antonelli@mi.infn.it
}

\section{Lino Miramonti}

Dipartimento di Fisica, Università degli Studi di Milano e I.N.F.N. Sezione di Milano, Via Celoria 16 - 20133 MIlano, Italy. E-mail:1ino.miramonti@mi.infn.it

\section{Carlos Peña-Garay}

Instituto de Fisica Corpuscular, CSIC-UVEG, Valencia 46071 Spain

E-mail:penagaray@gmail.com

\section{Aldo Serenelli}

Instituto de Ciencias del Espacio (CSIC-IEEC), Facultad de Ciències, Campus UAB, 08193

Bellaterra, Spain E-mail:aldosealiga.ieec.uab.es

\begin{abstract}
Solar neutrinos contributed significantly to the development of elementary particle physics and astrophysics. After the results that at the turn of millennium solved the Solar Neutrino Puzzle, proving that neutrinos are massive and oscillating particles, in the last decade solar neutrino experiments and KamLAND went on producing a great amount of data. Meanwhile, with the advent of Borexino experiment, it was possible for the first time to perform a real time measurement of the medium and low energy part of the $p p$ chain spectrum and to put limits on the CNO flux. We revise all of these results and the parallel phenomenological analyses, discussing the emerging general picture of mixing and masses and the impact on solar models. We focus in particular on the main open issues, like the study of low and medium energy part of the spectrum (and the potential anomalies that seem to emerge) and the solar metallicity problem. We discuss the potentialities of different future experiments already approved or under discussion, like SNO+, LENA and the future generation of liquid scintillators.
\end{abstract}

XV Workshop on Neutrino Telescopes,

11-15 March 2013

Venice, Italy

\footnotetext{
* Speaker.

†ne of us, V. A., would like to thank the organizers of the "Neutrino Telescopes" workshop for the opportunity given to him and the stimulating human and scientific atmosphere of the conference.
} 
Most of neutrino properties, of great impact on elementary particle physics and astrophysics, were discovered by studying natural sources (the Sun, the atmosphere and the SuperNovas). Solar neutrinos caught the interest of the scientific community for many years until the "annus mirabilis" 2002, when SNO and KamLAND, in conjunction with the data from previous experiments, found the final solution of the solar neutrino puzzle [1]. In the following decade the neutrino community turned its attention mainly to the study of artificial sources. Reactor neutrino experiments [2] proved that the mixing angle between the $1^{\text {st }}$ and the $3^{\text {rd }}$ generation is different from zero, confirming the hints from accelerator neutrino experiments [3] and from global phenomenological analyses, thus completing the mixing pattern and enforcing the hope of a future search of CP violation in the leptonic sector. The already cited accelerator experiments and the ones studying the CNGS beam [4], contributed to improve the determination of the "atmospheric" oscillation parameters and found the first appearance signals in a neutrino beam. However, it is worthwhile to remember that also during these years solar neutrino experiments went on producing data and new analyses were performed. Their aim was not anymore to prove the existence of neutrino mass and oscillations, but to improve the determination of the mass and mixing parameters, look for possible anomalies and test the medium and low energy part of the spectrum, almost unexplored till a few years ago. These studies are fundamental to get a better insight in the oscillation pattern (relevant for elementary particle models) and even more for astrophysics, trying to solve the metallicity problem and discriminate between different versions of the Solar Standard Models (SSM) [5, 6].

The SNO collaboration recently performed a combined analysis [7] of all the data collected by the experiment: SNO-I and SNO-II (the '01-'03 salt phase), already combined by the LETA analysis, and SNO-III ('03-'06 data with ${ }^{3} \mathrm{He}$ filled proportional chambers). Unfortunately the value, $\Phi_{8_{\mathrm{B}}}=5.25 \pm 0.16$ (stat.) ${ }_{-0.11}^{+0.13}$ (syst.) $\times 10^{6} \mathrm{~cm}^{-2} \mathrm{~s}^{-1}$, recovered for ${ }^{8} \mathrm{~B}$ flux is in the middle between high Z [5] and low Z [6] SSM predictions and, therefore, it doesn't allow the discrimination between these models. The day-night (D-N) asymmetry compatible with zero and the absence of anomalous seasonal variations are compatible with the LMA (Large Mixing Angle) oscillation pattern, which is confirmed also by the data collected by SuperKamiokande in its phases of working, SK-II ('02-'05), SK-III ('06-'08) and SK-IV, started in 2008. The first analysis of SK-IV data [8] seems to indicate a D-N asymmetry different from zero at $2.7 \sigma$ (compatible with earth regeneration) and found also the expected spectrum distortion at energies lower than $6 \mathrm{MeV}$. In case of confirmation, this indication, not yet supported by a robust statistics (only $1 \sigma$ at the moment), would represent an important validation of the LMA oscillation mechanism also in the transition region between the vacuum dominated and the matter enhanced part of the spectrum, where other experiments seemed to find some anomalies. An independent confirmation of the oscillation solution came by the 2004-07 data of the reactor experiment KamLAND, which confirmed with an enlarged fiducial volume and reduced systematic uncertainty the 2002-04 results. KamLAND (KL) results restricts the allowed region in the oscillation parameter space and are in general agreement with the solar data, with a slight tension toward higher values of $\Delta m_{21}^{2}$ and $\tan ^{2} \theta_{12}$, which is anyhow reduced in a 3 flavour analysis. The values obtained by SNO collaboration in a global analysis of solar and KL data were the following [7]: $\Delta m_{21}^{2}=7.41_{-0.19}^{+0.21} \times 10^{-5} \mathrm{eV}^{2} ; \tan ^{2} \theta_{12}=0.446_{-0.029}^{+0.030}$; $\sin ^{2} \theta_{13}=2.5_{-1.5}^{+1.8} \times 10^{-2}$. SNO was fundamental to determine the mixing parameters (especially $\theta_{12}$ ), but this experiment alone could not exclude the LOW solution and this ambiguity is solved in favor of the LMA solution only including in the analysis the results from Borexino and previous 
solar experiments or the ones from KamLAND. The results of SNO collaboration's global analysis agrees with the ones obtained in 2011 by KL collaboration [9] and with the global phenomenological analyses presented in 2012 by various groups [10].

The real breakthrough of solar neutrino physics in the last years was the advent of Borexino, that started the real time study of the low energy neutrino spectrum. Previous experiments could investigate only the region above $4-5 \mathrm{MeV}$ and up to six years ago most of the $p p$ chain spectrum had been studied only by the radiochemical experiments, with measurements integrated over the energy. Thanks to the very high radiopurity reached and the further reduction, with the 2009 "calibration campaign", of systematic uncertainties, Borexino was able to measure accurately the $0.862 \mathrm{MeV}{ }^{7} \mathrm{Be}$ line and found the flux value [11] $\phi_{7}{ }_{B e}=3.10 \pm 0.15 \times 10^{9} \mathrm{~cm}^{-2} \mathrm{~s}^{-1}$, excluding the no-oscillation hypothesis at more than $5 \sigma$ and confirming the LMA solution. Borexino also measured the ${ }^{8} \mathrm{~B}$ contribution, with a low energy threshold ( $3 \mathrm{MeV}$ for the recoil electron), and found no evidence of D-N asymmetry. Unfortunately, also the ${ }^{7} \mathrm{Be}$ flux fall in the middle between the two values predicted by the high Z and low Z SSM and it is compatible with both of them, as it was in the case of ${ }^{8} \mathrm{~B}$ flux; hence experimental data cannot discriminate between these two versions of the model. To solve this ambiguity, future experiments will try to determine more accurately these fluxes and possibly to combine these information with the study of neutrinos from CNO cycle (see $[11,1,12]$ ), whose measurement would be essential also to test the properties of different stars in various evolution phases and could require the adoption of ad hoc experimental expedients, like the one suggested in [13]. In the SSM the pep neutrino flux, directly linked to the $p p$ one and strongly constrained by solar luminosity, is predicted with a small theoretical uncertainty and its accurate measurement would be a stringent test of the model. However, until 2011 no direct detection of these fluxes was available, due to the technical difficulty of these low energy measurements. The background reduction for pep neutrinos is even more challenging than in ${ }^{7} \mathrm{Be}$ case (due to the lower rates) and it required the adoption of a special technique, called TFC (Three Folder Coincidence), to suppress the main background source, the $\beta^{+}$emitter ${ }^{11} \mathrm{C}$. Borexino measured the pep flux value $\phi_{\text {pep }}=(1.6 \pm 0.3) \times 10^{8} \mathrm{~cm}^{-2} \mathrm{~s}^{-1}$ and found for CNO neutrinos the limit $\phi_{C N O}<7.710^{8} \mathrm{~cm}^{-2} \mathrm{~s}^{-1}$.

A further improvement could come from the SNO+ [14] experiment at SNO-LAB, where a liquid scintillator is going to replace the deuterium Čerenkov detector. Taking advantage of a larger mass and a position almost twice deeper than the Borexino one, SNO+ should have a better signal to background ratio and it hopes to reach a 5\% accuracy on pep rate. It is also expected to detect the low energy ${ }^{8} \mathrm{~B}$ neutrinos and above all the $\mathrm{CNO}$ ones. The future hopes of solar neutrino physics rely not anymore on Čerenkov detectors (limited at low energies by the low photon yield), nor on the radiochemical experiments (measuring only integrated rates), but, instead, they rest upon the possibilities of the next generation of scintillators. These multipurpose devices, planned to search for neutrinoless double decay and dark matter and characterized by large masses and high radiopurity, are ideal to perform low energy neutrino spectroscopy, extracting very low signals from background. A first example is given by LENA[15] (a 50 ktons organic scintillator) that will be hosted in the Physalmi underground laboratories and should be able also to study the ${ }^{7} \mathrm{Be}$ signal modulation, detect the low energy ${ }^{8} B$ neutrinos and attack the problem of pep and $\mathrm{CNO}$ measurement. Other future liquid scintillator experiments plan to use noble gases[16]. CLEAN/DEAP (using liquid Neon) at SNO-LAB and XMASS (a liquid Xenon detector) 
at Kamioka should have a mass of the order of tens of tons and plan to measure the ${ }^{7} \mathrm{Be}$ component, improve the $\theta_{12}$ determination, testing the stability of LMA solution, and try to measure the $p p$ component with a $1 \%$ and the $\mathrm{CNO}$ flux with a $10-15 \%$ accuracy. A contribution should come also by projects (like the one of the EU-US working group DARWIN) finalized to dark matter searches by means of LAr or LXe detectors, which will study the low energy $v-e^{-}$elastic scattering as one of the main background sources. A different strategy will be adopted by experiments[17] planning to study the lower $p p$ cycle components by means of inverse $\beta$ decay on metals like Indium, in the case of LENS and IPNOS, or Molybdenum (in the case of MOON).

\section{References}

[1] For a discussion on solar neutrino history see, for instance: V. Antonelli, L. Miramonti, C. Peña Garay, A. Serenelli, Adv. High Energy Phys. 2013 (2013) 351926; V. Antonelli and L. Miramonti Int. J. Mod. Phys. E 22 (2013) 1330009 and the references therein.

[2] Y. Abe et al. [DOUBLE-CHOOZ Collaboration], Phys. Rev. Lett. 108 (2012) 131801; F. P. An et al. [DAYA-BAY Collaboration], Phys. Rev. Lett. 108 (2012) 171803; J. K. Ahn et al. [RENO Collaboration], Phys. Rev. Lett. 108 (2012) 191802.

[3] K. Abe et al. [T2K Collaboration], Phys. Rev. Lett. 107 (2011) 041801; P. Adamson et al. [MINOS Collaboration], Phys. Rev. Lett. 107 (2011) 181802.

[4] N. Agafonova et al. [OPERA Collaboration], JHEP 1311(2013)036; C. Rubbia, M. Antonello, et al. [ICARUS Collaboration], JINST 6 (2011) P07011 and Eur. Phys. J. C 73 (2013) 2599.

[5] N. Grevesse and A. J. Sauval, Space Sci. Rev. 85, 161 (1998); A. M. Serenelli, W. C. Haxton and C. Pena-Garay, Astrophys. J. 743 (2011) 24.

[6] A. Serenelli, S. Basu, J. W. Ferguson and M. Asplund, Astrophys. J. 705 (2009) L123; M. Asplund, N. Grevesse, A. J. Sauval and P. Scott, Ann. Rev. Astron. Astrophys. 47 (2009) 481.

[7] B. Aharmim et al. [SNO Collaboration], Phys. Rev. C 88 (2013) 025501.

[8] H. Sekiya [for the Super-Kamiokande Collaboration], arXiv:1307.3686 [astro-ph.SR].

[9] A. Gando et al. [KamLAND Collaboration], Phys. Rev. D 83 (2011) 052002.

[10] G. L. Fogli, E. Lisi, A. Marrone, D. Montanino, A. Palazzo and A. M. Rotunno, Phys. Rev. D 86 (2012) 013012; D. V. Forero, M. Tortola and J. W. F. Valle, Phys. Rev. D 86 (2012) 073012; M. C. Gonzalez-Garcia, M. Maltoni, J. Salvado and T. Schwetz, JHEP 1212 (2012) 123.

[11] G. Bellini, J. Benziger,D. Bick, S. Bonetti, G. Bonfini,M. Buizza Avanzini,B. Caccianiga, L. Cadonati et al., Phys. Rev. Lett. 107 (2011) 141302; G. Bellini et al. [Borex. Collab.], arXiv:1308.0443[hep-ex].

[12] V. Antonelli, L. Miramonti, will appear on the Proceedings of the "14th ICATPP Conference", Villa Olmo (Como), 23-27 September 2013 (arXiv:1311.2575 [astro-ph.SR]).

[13] F. L. Villante, A. Ianni, F. Lombardi, G. Pagliaroli and F. Vissani, Phys. Lett. B 701 (2011) 336.

[14] S. J. M. Peeters [SNO+ Collaboration], Nucl. Phys. Proc. Suppl. 237-238 (2013) 98.

[15] M. Wurm et al. [LENA Collaboration], Astropart. Phys. 35 (2012) 685.

[16] M. Kos [DEAP/CLEAN Collaboration], PoS ICHEP 2010 (2010) 455; H. Sekiya, J. Phys. Conf. Ser. 308 (2011) 012011.

[17] C. Grieb, J. Link and R. S. Raghavan, Phys. Rev. D 75, 093006 (2007); Y. Fukuda and S. Moriyama, J. Phys. Conf. Ser. 375 (2012) 042054; M. Nomachi, et al., Nucl. Phys. Proc. Suppl. 138 (2005) 221. 